\title{
Detection of the dietary xenoglycan N-glycolylneuraminic acid (Neu5Gc) and anti-Neu5Gc antibodies within reproductive tracts of male and female infertility subjects
}

\author{
Julie M Sroga ${ }^{1 \#}$, Diana H Wu ${ }^{2 \#}$, Fang Ma ${ }^{3 \#}$, EillenTecle ${ }^{4}$, Hector Sequoyah Reynoso ${ }^{4}$, Ilana B. Ressler ${ }^{1}$, Rose Maxwell ${ }^{5}$, Rachael Ferrari ${ }^{5}$, Leah \\ Whigham $^{6}$, Pascal Gagneux ${ }^{4 *}$ and Steven R. Lindheim ${ }^{5 *}$ \\ ${ }^{1}$ Division of Reproductive Endocrinology and Infertility, Department of OB/GYN, University of Cincinnati Medical Center, Cincinnati, Ohio, USA \\ ${ }^{2}$ Division of Reproductive Endocrinology and Infertility, Department of OB/GYN, Oregon Health and Science University, Portland, Oregon, USA \\ ${ }^{3}$ West China Medical Center of Sichuan University, China \\ ${ }^{4}$ Department of Pathology, University of California San Diego, San Diego, California, USA \\ ${ }^{5}$ Department of Obstetrics and Gynecology, Wright State University, Boonshoft School of Medicine, Dayton, Ohio, USA \\ ${ }^{6}$ Paso del Norte Institute for Healthy Living, El Paso, Texas, USA
}

\begin{abstract}
Objective: To assess the frequency of dietary xenoglycanNeu5Gc and antibodies in males and females and its impact on fertility.

Design: Prospective study of semen, uterine lavage, and follicular fluid from subjects undergoing infertility evaluation or in vitro fertilization (IVF) and fertile controls. Setting: University based infertility program.

Participants: Males ( $\mathrm{n}=23)$ and females ( $\mathrm{n}=27)$ undergoing semen analysis and saline infusion sonography as part of their diagnostic evaluation and 37 women undergoing IVF were compared to fertile male $(\mathrm{n}=15)$ and female $(\mathrm{n}=14)$ controls.

Intervention: Neu5Gc was measured by affinity purified antibody staining on Western blots, flow cytometry, and by high performance liquid chromatography. AntiNeu5Gc antibodies were determined by ELISA.

Main parameters measured: Frequency and levels of Neu5Gc antigen within sperm and endometrial cells and antibodies in semen, uterine lavage, and follicular fluid. Semen quality and IVF outcomes were assessed between antigen and antibody positive and negative subjects.

Results: In infertile subjects,Neu5Gc was detected in $26 \%$ of sperm and $54 \%$ of endometrial cells compared to $0 \%$ in male and $0 \%$ female controls. Anti-Neu5Gc antibodies were identified in $54 \%$ of seminal fluid, $41 \%$ in uterine lavage and $43 \%$ of follicular fluid samples. There were no differences in semen parameters, oocyte quality, and embryo development in the presence or absence of Neu5Gc antigen or antibody. However, clinical pregnancy rate was significantly lower in the presence of anti-Neu5Gc antibodies intrauterine lavage ( $0 \%$ vs. 54.5.0\%, $\mathrm{p}<0.05)$.
\end{abstract}

Conclusions: Neu5Gc and directed antibodies are present in reproductive tracts of both male and female infertility subjects. Our results suggest their presence may interfere with fertility within the uterine environment.

Abbreviations: 1,2-diamino-4,5-methylenedeoxybenzene (DMB), assisted reproductive technologies (ART), clinical pregnancy rate (CPR), cytidine monophosphate N-Acetylneuraminic acid hydroxylase (CMAH), embryo transfer (ET), high performance liquid chromatography (HPLC), in vitro fertilization (IVF), $\mathrm{N}$-glycolylneuraminic Acid (Neu5Gc).

\section{Introduction}

All cell surfaces are coated by a complex array of glycans. In mammals, the majority of these surface glycans are capped with sialic acids, nine carbon backbone acidic amino sugars. By virtue of their position and high abundance at the outermost cell surface, sialic acids are involved in multiple functions including cellular recognition during development, infection and immune processes [1]. The role of sialic acids in reproduction is not fully understood, but acquisition of a
Correspondence to: Steven R. Lindheim, MD, MMM, Department of Obstetrics and Gynecology, 128 E. Apple Street, Suite 3811 Dayton, OH 45409, USA, Tel 937.208.2850; Fax 937.222.725, E-mail: steven.lindheim@wright.edu

Pascal Gagneux, PhD, Department of Pathology, 9500 Gilman Drive, La Jolla, California, 92093-0687, USA, Tel: 858.822.3040, E-mail: pgagneux@ucsd.edu

"These authors contributed equally to the study

Key words: infertility, N-glycolylneuraminic Acid,sialic acid, xenoglycan, xenosialitis

Received: September 26, 2015; Accepted: October 23, 2015; Published: October 27, 2015

Funding: G. Harold and Leila Y. Mathers Charitable Foundation grant 
Sroga JM (2015) Detection of the dietary xenoglycan N-glycolylneuraminic acid (Neu5Gc) and anti-Neu5Gc antibodies within reproductive tracts of male and female infertility subjects

sialylated glycocalyx is crucial to sperm function [2]. Most recently, it has been shown that sperm sialidases are involved in desialylation during capacitation [3] and that sialic acids of sialyl Lewis X glycans on human egg zona pelucida are essential for sperm binding [4]. Implantation also involves L-selectins on the blastocyst known to interact with sialic acid containing ligands on the endometrium $[5,6]$. The two most common sialic acids in mammals are $\mathrm{N}$-glycolylneuraminic acid (Neu5Gc) and $\mathrm{N}$-acetylneuraminic acid (Neu5Ac) [1]. Most mammals have an abundance of each molecule in different ratios on various cell surfaces. In contrast, humans have lost the ability to convert Neu5Ac to Neu5Gc due to a loss-of-function mutation in the cytidine monophosphate $\mathrm{N}-$ acetylneuraminic acid hydroxylase(CMAH)gene encoding the CMAH enzyme [7]. As a result, humans lack glycans capped by endogenous Neu5Gc on their cell surfaces and have an excess of Neu5Ac [8], a difference in hundreds of millions of molecules per cell. Despite the inability to endogenously produce Neu5Gc, dietary intake of red meat and dairy products, the richest sources of Neu5Gc, can result in accumulation and incorporation of trace amounts of Neu5Gc into the glycocalyx on human cells [9]. This has been reported in both human tumors such as breast, colon, and skin cancers [10] and normal tissues including secretory epithelium and blood vessels [9]. Moreover, most humans have circulating anti-Neu5Gc antibodies $[9,11]$ targeting this xenoglycan in serum $[9,12]$. Levels of anti-Neu5Gc antibodies vary among different individuals, but it has been suggested that chronic exposure to Neu5Gc and incorporation of this xenoglycan combined with circulating anti-Neu5Gc antibodies can promote chronic inflammatory states contributing to various diseases [14] including some of the diseases associated Hanganutziu-Deicher antibodies, which are reactive against Neu5Gc $[13,14]$. There is also experimental evidence for Neu5Gc acting as a xeno-auto antigen capable of exacerbating an inflammatory reaction within vasculature endothelium [15]. A recent study proved that the dietary xenoglycan Neu5Gc causes increased cancer via systemic inflammation in a mouse model [16]. More sensitive detection methods have provided evidence for widespread anti-Neu5Gc antibodies in most humans tested, including IgM, IgA, and IgG antibodies [17]. There is mounting evidence that the dietary bioaccumulation of xenoglycan and production of targeting antibodies might underlie various forms of xenosialitis [18].
We are unaware of any information on the presence of the xenoglycan Neu5Gc and anti-Neu5Gc antibodies in the human reproductive tract or of their potential consequences on fertility. The female reproductive tract through which sperm must traverse and in which embryos must implant contains antibodies originating from plasma ( $\operatorname{IgG})$ and locally produced ( $\operatorname{IgA}$ ) antibodies [19]. Inflammation and the resulting abnormal milieu is a possible mechanism for impaired implantation as seen in certain disease states (i.e. endometriosis, auto-immune diseases, tubal infertility) [20,21]. It has recently been suggested, that the loss of Neu5Gc may have caused reproductive incompatibility in ancestral hominin populations due to sexual selection by female immunity against the xenoglycan Neu5Gc on sperm or implanting blastocysts [22]. In a murine model, Cmah null females with antibodies against Neu5Gc have reduced fertility when mated with Neu5Gc positive wild-type males implicating that female immunity can result in reproductive incompatibility [22].

Thus, our objectives were 1) to determine if Neu5Gc antigen and targeting antibodies are present within the human reproductive tracts of males and females; 2) to compare levels between patients seeking infertility evaluation and fertile controls; 3 ) to assess whether antigen or antibody presence in the reproductive tract had any impact on semen quality and in-vitro fertilzation (IVF) outcomes, and 4) to investigate if they were associated with dietary intake of foods containing the antigenic Neu5Gc xenoglycan.

\section{Materials and methods}

This study was reviewed and approved by the Christ Hospital Institutional Review Board (Cincinnati, $\mathrm{OH}$ ) and the study was in compliance with privacy-act guidelines.

\section{Patient population}

From July 2010 to August 2014, subjects were recruited to participate in this prospective clinical study. Subjects enrolled were 1) those undergoing diagnostic infertility testing including a semen analysis $(n=23)$ and saline infusion sonography $(n=27) ; 2)$ patients undergoing in vitro fertilization (IVF) cycles $(n=37,20$ of which underwent a uterine lavage after a saline infusion sonography) and 3) fertile male

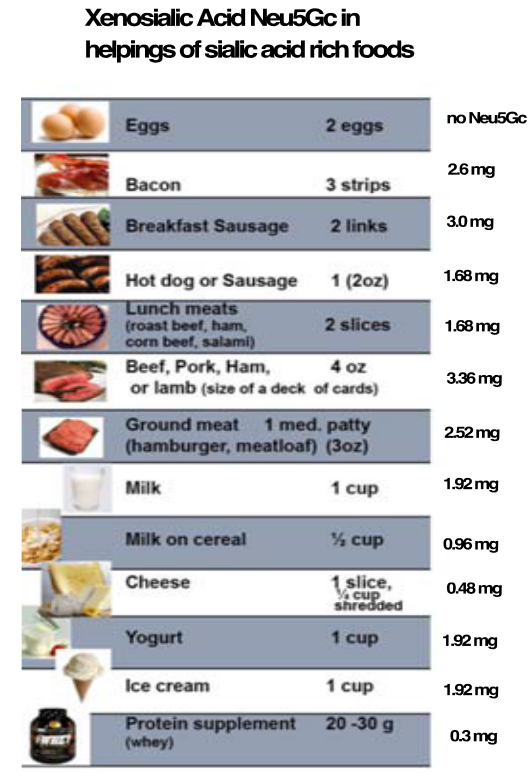

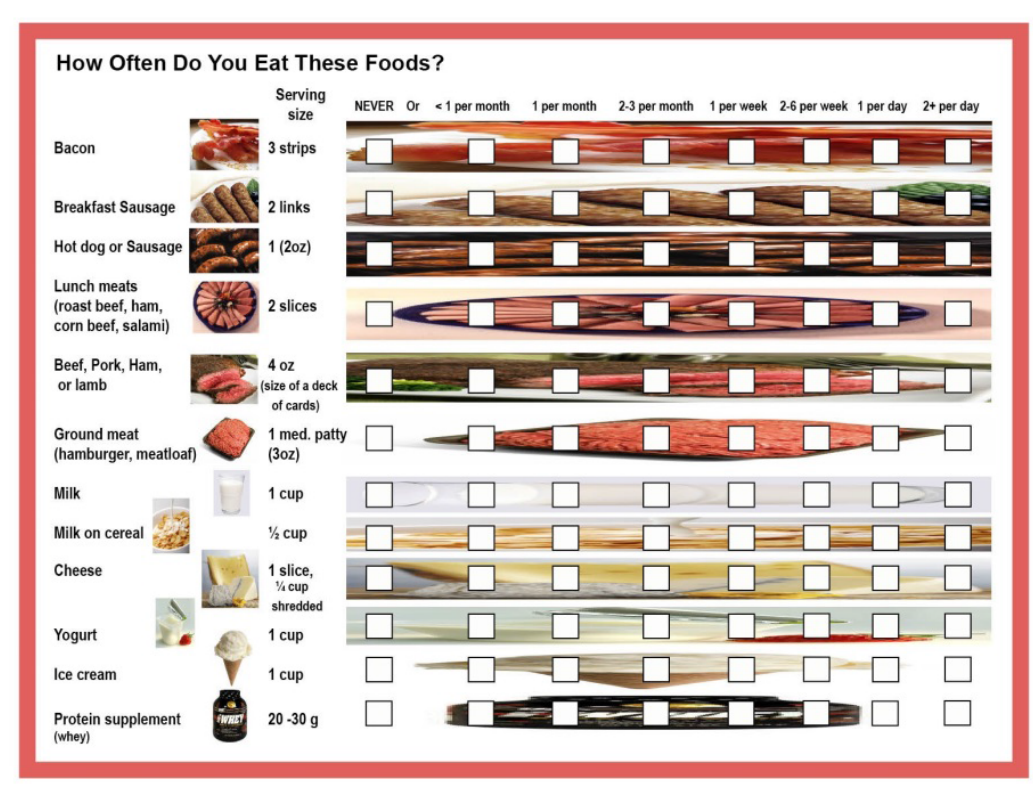

Figure 1. Dietary Questionnaire. 
Sroga JM (2015) Detection of the dietary xenoglycan N-glycolylneuraminic acid (Neu5Gc) and anti-Neu5Gc antibodies within reproductive tracts of male and female infertility subjects

$(\mathrm{n}=15)$ and female oocyte donors $(\mathrm{n}=14)$. Written informed consent was obtained from each subject candidate. Men between the ages of 18-50 years without previous vasectomy were eligible to participate.

Subjects meeting inclusion criteria were given a dietary questionnaire specifically designed for this study (Figure 1) including frequency of meat, dairy, and protein supplement intake as well as formula use as an infant, all of which contain the xenoglycan Neu5Gc. Xenoglycan load based on the questionnaire were calculated by using values from Tangvoranuntakul et al. [9].

\section{Sample acquisition}

Seminal fluid and sperm samples: Semen was collected on site in a sterile plastic specimen cup after a recommended period of abstinence of 2-5 days. The specimens were allowed to liquefy at $37^{\circ} \mathrm{C}$ for 30 minutes and analyzed within one hour of collection time for semen quality parameters and motion characteristics. Semen samples were then centrifuged twice for 8 minutes each time at $300 \mathrm{xg}$ (1200 RPM), and the supernatant (seminal fluid) was isolated and frozen at $-20^{\circ} \mathrm{C}$ before sample analysis. The remaining sperm pellet was reconstituted with 1-2 $\mathrm{mL}$ of phosphate buffered saline (PBS), then frozen at $-20^{\circ} \mathrm{C}$ for later analysis.

Uterine samples: Saline infusion sonography was performed either during the follicular phase of a spontaneous menstrual cycle or following a progestin withdrawal bleed. Following speculum examination and cervical cleansing, a 2 lumen 5 French balloon catheter (Cooper Surgical Inc. Trumbull, CT) flushed with sterile saline was placed into the lower uterine segment followed by instillation of sterile saline through the catheter until an adequate evaluation of the uterine cavity was obtained. At completion of the saline infusion sonography, fluid was aspirated collecting both endometrial cells and saline (mean volume $\sim 2 \mathrm{ml}$ ) for uterine lavage specimens. Uterine specimen samples were then centrifuged 15 minutes at $300 \mathrm{xg}(1200 \mathrm{RPM})$, and the supernatant was isolated from the remaining pellet (endometrial cells). Both samples were frozen at $-20^{\circ} \mathrm{C}$ until sample analysis.

\section{Assisted reproduction}

Ovarian stimulation-oocyte retrieval: Prior to ovulation induction for IVF, all female partners had normal uterine cavities as demonstrated on saline infusion sonography and operative hysteroscopy to correct cavity pathology.

Subjects undergoing IVF used a GnRH antagonist protocol. Briefly, ovarian stimulation was started on cycle day 3 following an oral contraceptive pill withdrawal bleed using daily injections of 150 to $300 \mathrm{IU}$ of recombinant follicle stimulating hormone $(\mathrm{rFSH})$ [Follistim ${ }^{\circledR}$ AQ Cartridge; Merck \& Co., Inc., Whitehouse Station, NJ, USA]. All cycles were monitored by serum estradiol levels and transvaginal ultrasound and the dose was adjusted according to response. GnRHantagonist (Ganirelix, $0.25 \mathrm{mg}$, subcutaneously daily [Merck \& Co., Inc., Whitehouse Station, NJ, USA]) was begun when lead follicles were 12-14 mm. When $\geq 3$ follicles reached $18-20 \mathrm{~mm}$, urinary hCG 10,000 IU was given and was followed by transvaginal oocyte aspiration 36 hours later.

Follicular fluid and serum samples: Follicular fluid from the first dominant follicle was collected for evaluation. Follicular fluid samples were centrifuged similar to uterine lavage specimens. Granulosa cells were discarded and follicular fluid was stored at $-20^{\circ} \mathrm{C}$. Serum was collected around the time of hCG administration, and samples were centrifuged (same as uterine lavage), red blood cells discarded, and serum was similarly stored at $-20^{\circ} \mathrm{C}$ until processing.

IVF-ICSI-embryo quality grading, culture, and embryo transfer: Fertilization was performed on the day of retrieval with sperm from a partner or donor using conventional IVF or intracytoplasmic sperm injection (ICSI). Three to four hours after oocyte retrieval, fertilization was performed in IVC-1 media by either conventional insemination or by ICSI in the presence of male factor infertility. The following day, fertilization was confirmed by the presence of two pronuclei. All normally fertilized embryos were cultured sequentially in Vitrolife's G1.3 and G2.3 media, supplemented with 5\% protein. An embryo transfer was performed either three or five days after oocyte retrieval under ultrasound guidance. At least 4 good quality cleavage stage embryos were required to continue culture to the blastocyst stage. Day3 embryos were evaluated according to cell number and were graded according to blastomere uniformity and the degree of fragmentation. Day- 3 embryo quality was ranked as good if $>6$ cells, grade 2 (slight to moderately uneven cells and slight to moderate fragmentation). Two to three embryos with the highest cell number and best morphology were selected for embryo transfer. Surplus embryos were then transferred to IVC-3 blastocyst media, which were re-evaluated on days 5 and/or 6 for development into suitable blastocysts for cryopreservation [23].

Pregnancy confirmation: Pregnancy was confirmed by serial serum $\beta$-hCG levels 7 to 10 days following embryo transfer. Clinical pregnancies were confirmed with transvaginal ultrasonography by a gestational sac at 6 to 7 weeks. Hormonal supplementation was continued until 10 weeks gestation or until a negative serum $\beta$-hCG.

Neu5Gc antigen detection: Neu5Gc was detected using an affinity purified polyclonal IgY antibody raised in chickens[24]. Frozen sperm were thawed and washed $3 \mathrm{X}$ with PBS. For flow cytometry, thawed sperm were first blocked with $0.5 \%$ fish gelatin in PBS (PBSfg) for $30 \mathrm{~min}$ at RT, then incubated with anti-Neu5Gc IgY at 1:1000 in PBSfg for 30 min on ice, washed 3 times with PBSfg, incubated with secondary donkey-anti chicken Dy488 at 1:1000 in PBSfg for 30 min on ice, washed $3 \mathrm{X}$ with PBSfg and measured on a FACScalibur Flow cytometer. Controls included: mild periodate treatment $(2 \mathrm{mM}$ NaIO4), which truncates the side chain of sialic acid and confirms sialic acid dependence of $\mathrm{Ab}$ staining; inhibition of the $\mathrm{Ab}$ with $10 \mathrm{nM}$ polyNeu5Gc, and non-specific isotype control.

\section{Human sperm Neu5GC detection via Western Blot}

Frozen sperm samples from control fertile males were thawed and centrifuged (500 g, 5 minutes). The supernatant, containing seminal fluid, was aspirated, followed by two washing cycles characterized by addition of $1 \mathrm{~mL}$ of phosphate buffered saline (PBS), centrifugation (500 g, 5 minutes), and aspiration of the supernatant. The remaining pellet was resuspended in $100 \mathrm{uL}$ of PBS. Protein concentration was determined by BCA, then an equal amount of protein ( 18 or 30 ug per lane) was loaded on a $1.5 \mathrm{~mm} 7 \%$ SDS-PAGE gel and run until the dye front almost ran off the gel (20 minutes at $20 \mathrm{mAmps}$ per gel, $60-80$ minutes at $40 \mathrm{mAmps}$ per gel). Gels were washed with milliQ water, then transferred onto a polyvinyldene fluoride (PVDF) membrane using a Bio-Rad SemiDry Transfer Cell for 1 hour (100 mAmps per membrane). Membranes were washed (5 minutes) in PBS-T $(0.1 \%$ Tween), blocked for one hour at room temperature in $0.5 \%$ fish gelatin PBS-T. Membranes were then incubated in PBS-T with 1:10,000 antiNeu5GC chicken IgY overnight at $4^{\circ} \mathrm{C}$. Membranes were washed four times (7 minutes in PBS-T), followed by a secondary incubation in PBS-T with 1:10,000 LiCOR donkey a-chicken 800 antibody for 45 minutes at room temperature. Membranes were washed four more 
Sroga JM (2015) Detection of the dietary xenoglycan N-glycolylneuraminic acid (Neu5Gc) and anti-Neu5Gc antibodies within reproductive tracts of male and female infertility subjects

times (7 minutes in PBS-T) before being imaged on a LiCOR Imaging System.

For western blots, thawed sperm membrane proteins were extracted with lysis buffer and loaded on denaturing SDS PAGE. The gels were transferred to membranes and blocked with PBSfg overnight at $4^{\circ} \mathrm{C}$. Blots were incubated in 1:50,000 affinity purified chicken IgY in TBST, $0.5 \%$ fish gelatin (Sigma) and $2 \mathrm{mg} / \mathrm{ml}$ of purified bovine serum albumin (BSA). Blots were washed and incubated with secondary $\mathrm{HRP}$-conjugated donkey anti-chicken $\mathrm{Ab}$ for $1 \mathrm{~h}$ at RT. Blots were developed using luminescent substrate (Pierce, Thermo Fisher Scientific, Rockford IL).

Shed endometrial cells were harvested from the pellet of the centrifuged thawed uterine flush samples. The cells were washed $3 \mathrm{X}$ with ddH2O and then incubated in $2 \mathrm{M}$ acetic acid for total sialic acid release. Released sialic acids were analyzed by high performance liquid chromatography (HPLC) of 1,2-diamino-4,5-methylenedeoxybenzene (DMB) derivatizedsialic acid extracts obtained from shed epithelial cells after $2 \mathrm{M}$ acetic acid hydrolysis at $80^{\circ} \mathrm{C}$ for $3 \mathrm{hrs}$. Released sialic acids were filtered through microcon 10 columns, MW $10 \mathrm{KD}$ (Amicon Millipore, Billerica, MA) by centrifuging at $13,000 \mathrm{~g}$ for $20 \mathrm{~min}$, treated with mild base and derivatized in DMB for $2.5 \mathrm{~h}$ at $50^{\circ} \mathrm{C}$ in the dark prior to HPLC analysis. HPLC was performed over Varian C18 reverse phase column, under isocratic conditions in $83 \%$ water, $7 \%$ methanol and $8 \%$ acetonitrile at a flow rate of $0.9 \mathrm{ml} / \mathrm{min}$ over 50 min on a Hitachi HPLC $[25,26]$. sialic acid standards were from bovine submaxillarymucin as well as commercially available Neu5Ac (Nacalai, San Diego, CA) and Neu5Gc (Inalco, San Luis Obispo, CA).

\section{Anti-Neu5Gc antibody detection}

Antibody titers in seminal fluid, uterine lavage, follicular fluid, and serum were determined by ELISA using Neu5Gc- and Neu5Acpolyacrylamide beads as plated targets (Padler-Karavani et al., 2008). Sialic acid dependence of antibody binding was confirmed by mild periodate treatment to destroy the side chain of sialic acidsand controlled for by mock treatment without the premixed periodate and sodium borohydride quenching agent.

Additional control samples were tested for anti-Neu5Gc antibodies by plating pooled WT mouse sera free of any anti human IgG and IgA. Human IgG or IgA were coated at various concentrations for standards (Padler-Karavani et al., 2013). All samples were diluted in 1XPBS/ chicken albumin/ pooled CMAH ko mouse sera (free of antihumanIgG and IgA). All samples were diluted 100 fold, except for uterine samples which were diluted 10 fold. The secondary antibody used was AP goat anti human IgG or or AP goat anti human IgA.

\section{Clinical outcomes in fertility patients}

Outcomes measured included 1) The frequency of Neu5Gc Antigen in semen samples and EC and Neu5GC antibody in seminal fluid, uterine lavage and follicular fluid; 2) The effect of Neu5GC antigen or anti- Neu5GC antibodies on semen parameters (semen quality including volume, concentration, motility, and strict morphology) and IVF outcomes (i.e. number of retrieved oocytes, fertilized embryos, embryo quality, rates of blastulation, pregnancy rate (PR) and clinical pregnancy rate (CPR). Analyses were also performed to correlate Neu5GC antigen or anti- Neu5GC antibody presence in reproductive tissue with dietary intake.

\section{Statistics}

Statistical analyses were performed using the Statistical Package for the Social Sciences (SPSS version 18; SPSS, Inc., Chicago, IL). Student's t-test was utilized to compare means for parametric data between Neu5 Gc Ag and Ab positive and negative subjects. Chi-square was used for categorical comparisons. Pearson correlation was used for correlation analysis. Significance was assessed at $p<0.05$. Means expressed as \pm SEM.

\section{Results}

\section{Detection of Neu5Gc Antigen and Anti-Neu5Gc Antibodies}

Semen and sperm samples: Neu5Gc was identified in $26 \%$ $(6 / 23)$ of sperm samples from males undergoing fertility evaluation compared to $0 / 15$ male controls, $\mathrm{p}<0.05$. Anti-Neu5Gc antibodies in males undergoing fertility evaluation were detected in $54 \%$ of SF (8/13; insufficient sample in 10 patients). Specifically, $54 \%$ had IgA Abs detected, $38 \%$ had IgG and 38\% IgM, respectively. Antibodies were not measured in fertile male controls. (Figure 2 and Table 1).

Uterine lavage, follicular fluid, and serum: The presence of Neu5Gc in endometrial cells from female infertility patients was detected in 54\% (9/17; insufficient sample in 10 patients). Neu5Gc was not measured in fertile female controls. Anti-Neu5Gc antibodies were detected in $41 \%$ of uterine lavage $(11 / 27)$ from female infertility patients (specifically, 37\% IgA, 27\% IgG, and 0\% IgM) compared to none of the female controls $(0 \%, 0 / 14, \mathrm{p}<0.05)$ Anti-Neu5Gc antibodies were detected in $43 \%$ of follicular fluid (16/37), and $44 \%$ of serum ([14/32]; insufficient sample in 5 patients) (Table 2). Of the follicular fluid samples, $22 \%$ had IgA, 32\% IgG, and 19\% IgM while in serum samples, $33 \%$ had IgA, $60 \%$ IgG, and $0 \%$ IgM, respectively. Neu-5Gc antibodies in follicular fluid and serum were not measured in fertile
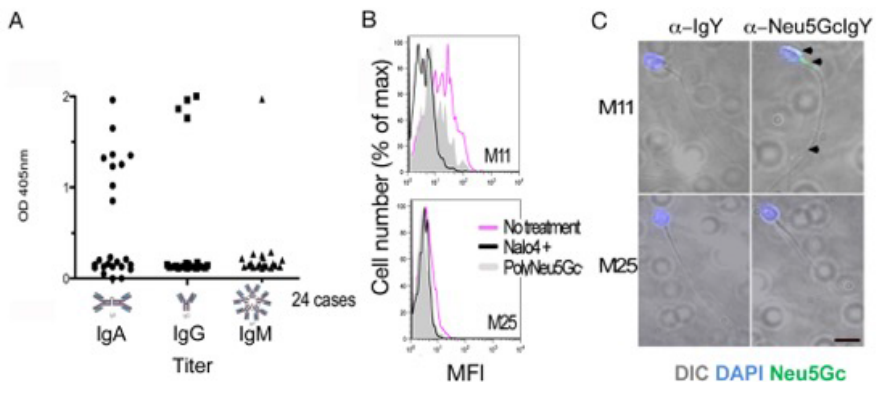

Figure 2. ELISA for Anti-Neu5Gc antibodies, flow cytometry and fluorescent microphotograph of sperm.

A) ELISA data for anti-Neu5Gc antibodies in seminal fluid from infertility patients. B) Flow cytometry on sperm cells from infertility patients showing Neu5Gc

positive (M11) and negative individual (M25), treatment by sodium periodate abrogates the side chain of sialic acid and reduces the signal and so does blocking

with poly Neu5Gc. C) fluorescent microphotograph of sperm positive and negative for Neu5Gc (green fluorescence for anti-Neu5Gc antibody indicated by black arrow heads, sperm nuclei stained blue with DAPI .

Table 1. Prevalence of Neu5Gc antigen and Anti-Neu5Gc antibody in semen of male subjects undergoing fertility evaluation compared to controls.

\begin{tabular}{|l|l|l|}
\hline & $\begin{array}{l}\text { Neu5 Gc Antigen in } \\
\text { Sperm Samples }\end{array}$ & $\begin{array}{l}\text { Neu5 Gc Antibody in } \\
\text { Seminal Fluid }\end{array}$ \\
\hline $\begin{array}{l}\text { Males Undergoing Fertility } \\
\text { Evaluation }\end{array}$ & $26 \%(6 / 23)^{*}$ & $54 \%(8 / 13) \dagger$ \\
\hline Male Controls & $0 \%(0 / 15)^{*}$ & Not measured \\
\hline $\begin{array}{l}* \\
\mathrm{p}<0.05\end{array}$ \\
$\dagger$ Insufficient seminal fluid sample in 10 patients
\end{tabular}


Sroga JM (2015) Detection of the dietary xenoglycan N-glycolylneuraminic acid (Neu5Gc) and anti-Neu5Gc antibodies within reproductive tracts of male and female infertility subjects

Table 2. Prevalence of Neu5Gc antigen and Anti-Neu5Gc antibodies in uterine lavage, folllicular fluid, and serum compared to controls.

\begin{tabular}{|l|c|c|c|}
\hline & $\begin{array}{c}\text { Uterine Lavage Neu5 } \\
\text { Gc Antigen }\end{array}$ & $\begin{array}{c}\text { Uterine Lavage } \\
\text { Neu5 Gc Antibody }\end{array}$ & $\begin{array}{c}\text { Follicular Fluid } \\
\text { Neu5 Gc Antibody }\end{array}$ \\
\hline $\begin{array}{l}\text { Females Undergoing Fertility } \\
\text { Evaluation }\end{array}$ & $54 \%(9 / 17)^{*}$ & $41 \%(11 / 27) \dagger$ & $43 \%(16 / 37)$ \\
\hline Female Controls & Not measured & $0 \%(0 / 14) \dagger$ & $44 \%(14 / 32)^{\ddagger}$ \\
\hline
\end{tabular}

"Insufficient follicular fluid sample in 10 patients

$\uparrow \mathrm{p}<0.05$

${ }^{\ddagger}$ Insufficient follicular fluid sample in 5 patients

female controls.

\section{Demographics}

Male Subjects: Demographics including age $(30.5 \pm 3.5$ years vs. $32.2 \pm 5.8$ years $)$ or BMI $\left(27.3 \pm 5.7 \mathrm{~kg} / \mathrm{m}^{2}\right.$ vs. $28.7 \pm 6.5 \mathrm{~kg} / \mathrm{m}^{2}$, p-NS) were similar between patients that were positive or negative for Neu5Gc antigen on sperm or targeting antibodies within SF (Table 3) and controls ( $31.4 \pm 8.5$ years and $\left.25.3 \pm 4.7 \mathrm{~kg} / \mathrm{m}^{2}\right)$.

Female subjects: Age ( $36.8 \pm 6.9$ years vs. $33.0 \pm 5.7$ years $)$, BMI $\left(29.1 \pm 5.7 \mathrm{~kg} / \mathrm{m}^{2}\right.$ vs. $\left.26.5 \pm 8.3 \mathrm{~kg} / \mathrm{m}^{2}, \mathrm{p}-\mathrm{NS}\right)$ or primary infertility diagnosis (data not shown) was not different between patients that were positive or negative for anti-Neu5Gc antibodies in uterine or ovarian environments (Table 3 ). While controls were significantly younger $(27.7 \pm 2.9$ years, $\mathrm{p}<0.05)$, BMI was similar $(25.2 \pm 4.7 \mathrm{~kg}$ / $\mathrm{m}^{2}$ ) to study subjects. Subjects who were positive for any antibody type (IgA, or IgG) within the uterine lavage had a lower gravidity $(1.5 \pm 1.7$ vs. $2.9 \pm 1.8, \mathrm{p}<0.05)$ and parity $(0.2 \pm 0.4$ vs. $1.3 \pm 1.5, \mathrm{p}<0.05)$ when compared to antibody negative subjects but not to controls $(1.4 \pm 0.4$ and $1.3 \pm 0.4$, and $1.3 \pm 0.4$, p-NS). No difference was seen in any demographic parameter and antibody presence within follicular fluid subjects undergoing IVF cycles.

\section{Diagnostic testing and clinical outcomes}

Semen analysis and saline infusion sonography: Overall, the presence of Neu5Gc antigen or anti-Neu5Gc antibodies did not correlate with differences in semen quality or the presence of uterine pathology. Specifically, semen volume $(3.0 \mathrm{~mL} v s .2 .7 \mathrm{~mL})$, total sperm concentration $\left(55.7 \times 10^{6} v s .42 .1 \times 10^{6}\right)$, motility $(54.2 \%$ vs. $57.9 \%)$ and morphology (5.5\% vs $6.4 \%)$, and intrauterine pathology identified on saline infusion sonography ( $18 \%$ vs. $38 \%)$ were similar regardless of Neu5Gc antigen or anti-Neu5Gc antibody status (Table 3).

Follicular fluid, uterine lavage and IVF outcomes: In those undergoing IVF, the total number of retrieved oocytes (10.7 \pm 5.5 vs. $11 \pm 5.9, \mathrm{p}-\mathrm{NS})$ and Metaphase II oocytes $(8.1 \pm 4.2$ vs. $8.1 \pm 4.0$, p-NS) were similar regardless of detectable anti-Neu5Gc antibodies within follicular fluid of infertility patients. There were no adverse effects of anti-Neu5Gc antibodies on high quality embryos and rates of blastulation (data not shown). PR and CPR of these 37 subjects were similar in follicular fluid anti-Neu5Gc antibody positive or negative patients, respectively ( $25.0 \%$ vs. $23.8 \%$, p-NS) and CPR $(18.8 \%$ vs. $23.8 \%, \mathrm{p}-\mathrm{NS}$ ).

Analysis was done on 20 of 37 IVF subjects who had uterine lavage with known Neu5Gc antigen and antibody status, The CPR per patient was significantly lower in those who were positive for any antibody $(0 \%$ vs. $54.5 \%, \mathrm{p}<0.05)$ and specifically for IgG antibody $(0 \%$ vs. $50 \%, \mathrm{p}<0.05)$, though not different in the presence or absence of IgA antibody ( $0 \%$ vs. $42.8 \%, \mathrm{p}=0.08)$. CPR per cycle (fresh and frozen combined cycles, $\mathrm{n}=27)$ was lower in the presence of any antibody $(0 \%$
Table 3. Comparison of demographics in Neu5Gc antigen positive and negative semen and endometrial cells subjects.

\begin{tabular}{|c|c|c|c|}
\hline & \multicolumn{2}{|c|}{ Sperm Neu5Gc } & \multirow[t]{2}{*}{ p-value } \\
\hline & + & - & \\
\hline Subjects & $\mathrm{n}=6$ & $\mathrm{n}=17$ & \\
\hline Age (years) & $30.5 \pm 3.5$ & $32.2 \pm 5.8$ & NS \\
\hline BMI $\left(\mathrm{kg} / \mathrm{m}^{2}\right)$ & $27.3 \pm 5.7$ & $28.7 \pm 6.5$ & NS \\
\hline Semen Volume (ml) & $3 \pm 1.6$ & $2.7 \pm 1.4$ & NS \\
\hline Sperm Conc. (million) & $55.7 \pm 62.7$ & $42.1 \pm 31.1$ & NS \\
\hline Total Motility (\%) & $54.2 \pm 20.1$ & $57.9 \pm 12.3$ & NS \\
\hline \multirow[t]{3}{*}{ Normal Morphology (\%) } & $5.5 \pm 6.1$ & $6.4 \pm 15.1$ & NS \\
\hline & \multicolumn{2}{|c|}{ Endometrial Cells Neu5Gc } & \\
\hline & + & - & \\
\hline Subjects & $\mathrm{n}=9$ & $\mathrm{n}=8$ & \\
\hline Age (years) & $36.8 \pm 6.9$ & $33 \pm 5.7$ & NS \\
\hline BMI $\left(\mathbf{k g} / \mathbf{m}^{2}\right)$ & $29.1 \pm 5.7$ & $26.5 \pm 8.3$ & NS \\
\hline Gravidity & $1.4 \pm 1.6$ & $1.5 \pm .6$ & NS \\
\hline Parity & $1 \pm 1.7$ & $0.3 \pm .5$ & NS \\
\hline Pathology on SIS (\%) & 18 & 38 & NS \\
\hline
\end{tabular}

vs $50 \%, \mathrm{p}<0.01)$, IgA antibody ( $0 \%$ vs $31.4 \%, \mathrm{p}<0.01)$; and IgG antibody ( $0 \%$ vs $46.1 \%, p<0.05)$ when compared to antibody negative subjects (Figures 3 and 4 ).

Dietary intake and correlation between serum and reproductive fluids: No positive correlation was noted between dietary intake of red meat, dairy products nor infant formula use and Neu5Gc or anti-Neu5Gc antibody status in any reproductive fluid or serum. The presence of any serum anti-Neu5Gc antibody positively correlated to antibody presence within follicular fluid $(r=0.58, \mathrm{p}<0.001)$.

\section{Discussion}

To our knowledge this study is the first to explore the frequency of incorporation of non-human xenoglycanNeu5Gc sialic acid and the potential role of Neu5Gc and targeting antibody in male and female infertility subjects. Our data demonstrate that Neu5Gc and targeting antibody are abundantly expressed within both the male and female reproductive tracts of infertility subjects. Our preliminary results also suggest that the presence of the Neu5Gc xenoantigen or anti-Neu5Gc antibodies do not adversely impact sperm parameters or ovarian function including oocyte development and embryo quality. However, the presence within the uterine environment may possibly impact implantation and pregnancy outcomes. 
A

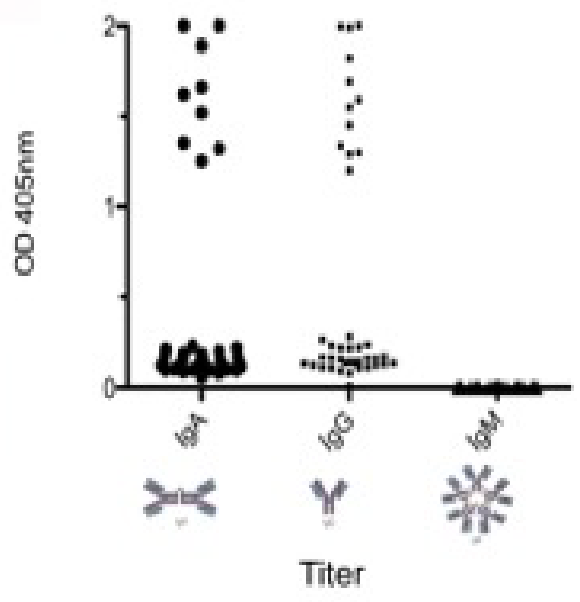

B

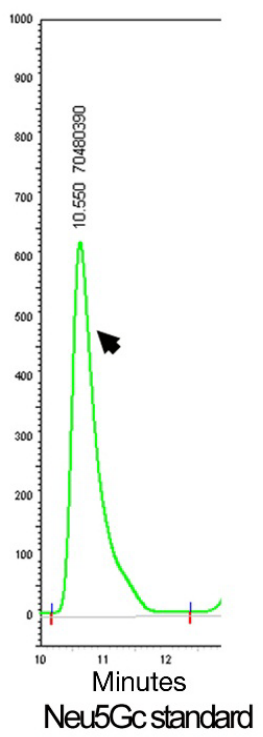

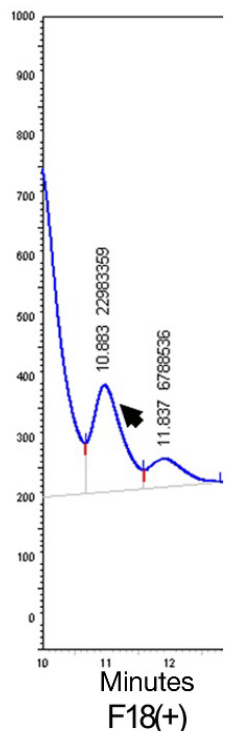

$\mathrm{F} 18(+)$

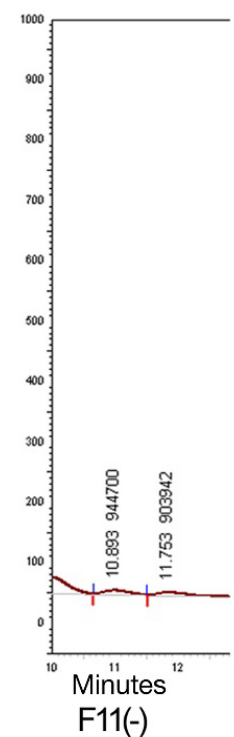

Figure 3. Uterine lavage anti-Neu5Gc antibodies by ELISA. A) Measurements of anti-Neu5Gc antibodies by ELISA in uterine lavage of infertility patients. B) Detection of Neu5Gc in uterine lavage (shed endometrial cells) by HPLC, standard, positive sample and negative sample

The presence of Neu5Gc antigen or anti-Neu5Gc antibodies is not surprising as other investigators have reported the presence of this xenoglycan and associated antibodies in both normal and diseased human tissues $[9,10]$. With respect to fertility, it has been suggested that antibodies within the uterine environment may impair fertility by two potential mechanisms including sperm inactivation and embryo rejection or failed implantation secondary to incompatibility between the blastocyst and receptive uterus [22]. Our results reveal a lower $\mathrm{CPR}$ in the presence of anti-Neu5Gc antibodies within the uterine environment. Moreover, none of the women positive for anti-Neu5Gc IgG antibodies achieved pregnancy even with repeat embryo transfer. In light of our study subjects undergoing IVF and the production of HQ embryos, it would suggest that implantation may be negatively affected in the presence of anti-Neu5Gc antibodies, contributing to infertility in these subjects.

The unique human sialic acids biology has brought about a situation where a dietary molecule can get incorporated into healthy human tissues and become a xeno-auto-antigen. The presence of the xeno-auto-antigen Neu5Gc in human reproductive tracts, cells, and secretions could contribute to anti-glycan immune related impaired fertility (xenosialitis affecting fertility). The presence of a non-self glycan (Neu5Gc) at the molecular frontier of the embryo, combined with antibodies targeting this molecule is consistent with our observed adverse effects on implantation success.

The fact that we found no consistent correlations between the dietary factors and antigen or antibody status is not surprising. The accumulation of xenoglycan Neu5Gc is highly variable between individuals and between tissues within individuals [9]. Additionally, dietary intake over a lifetime is challenging to assess accurately and would require very strong associations to detect significant correlations with our current intake assessment methodology. For future studies to better address the relationship between dietary load of xenoglycans and the biological consequences, extensive resources will need to be
A

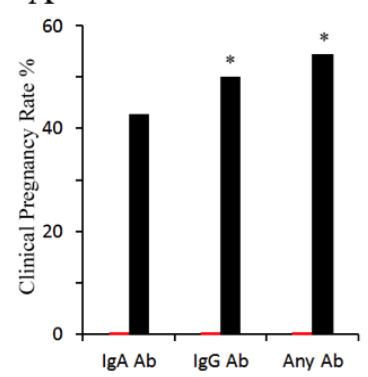

B

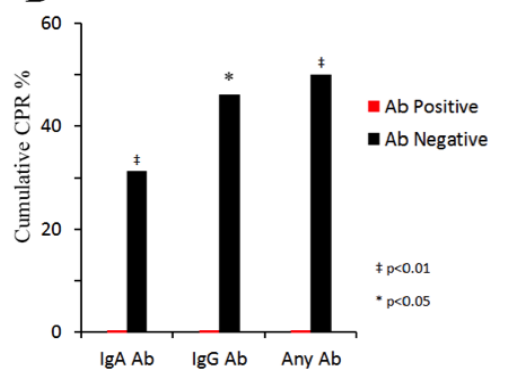

${ }^{*} \mathrm{p}<0.05 ; \dagger \mathrm{p}<0.01$.

Figure 4. Pregnancy and clinical pregnancy rates in IVF subjects positive and negative for anti-Neu5Gc antibodies $(\mathrm{Ab})$ in uterine lavage.

devoted to either collecting dietary intake prospectively over long periods of time or developing detailed recall questionnaires specifically targeting dietary sources of Neu5Gc.

Our study demonstrates that Neu5Gc and targeting antibody are abundant within the human reproductive tract. To our knowledge, this is the first study to evaluate the role of this xenoglycan and targeting antibodies within human fertility. However, there are a number of limitations including: 1) small sample size which was not powered to detect clinical outcomes; 2) fluids collected were not all from same cohort (semen-uterine lavage-follicular fluid-serum); and 3) the precise localization of Neu5Gc antigen in the uterus could not be established beyond its presence on shed epithelial cells recovered from the uterine lavage.

Future studies will focus on elucidating the potential functional deficits of sperm bearing Neu5Gc and targeting antibodies and 
Sroga JM (2015) Detection of the dietary xenoglycan N-glycolylneuraminic acid (Neu5Gc) and anti-Neu5Gc antibodies within reproductive tracts of male and female infertility subjects

evaluating the male and female systems within the same couple in order to better understand the role of Neu5Gc and targeting antibodies in subfertile or infertile couples. Such investigations will reveal to what extent xenosialitis, the contribution of the xenoglycan Neu5Gc to heightened or chronic inflammation, also affects human reproduction [18] and will point to potential mechanisms involved.

\section{Acknowledgments}

Authors would like to thank Dr. Michael Thomas and Dr. Krystene DiPaola at the University of Cincinnati for patient referral for participation and the G. Harold and Leila Y. Mathers Foundation for funding for the Gagneux laboratory.

\section{References}

1. Varki A, Schauer R (2009) Sialic Acids. Edn 2. Varki A, Cummings R, Esko J, Freeze H, Stanley P, Bertozzi C, Hart G, Etzler M editors, Essentials of Glycobiology. Plainview, NY. Cold Spring Harbor Press 199-218.

2. Schröter S, Osterhoff C, McArdle W, Ivell R (1999) Theglycocalyx of the sperm surface. Hum Reprod Update 5: 302-313. [Crossref]

3. Ma F, Wu D, Deng L, Secrest P, Zhao J, et al. (2012) Sialidases on mammalian sperm mediate deciduous sialylation during capacitation. J BiolChem 287: 38073-38079. [Crossref]

4. Pang PC, Chiu PC, Lee CL, Chang LY, Panico M, et al. (2011) Human sperm binding is mediated by the sialyl-Lewis(x) oligosaccharide on the zonapellucida. Science 333: 1761-1764. [Crossref]

5. Genbacev OD, Prakobphol A, Foulk RA, Krtolica AR, Ilic D, et al. (2003) Trophoblast L-selectin-mediated adhesion at the maternal-fetal interface. Science 299: 405-408. [Crossref]

6. Margarit L, Gonzalez D, Lewis PD, Hopkins L, Davies C, et al. (2009) L-Selectin ligands in human endometrium: comparison of fertile and infertile subjects. Hum Reprod 24: 2767-2777. [Crossref]

7. Chou HH, Takematsu H, Diaz S, Iber J, Nickerson E, et al. (1998) A mutation in human CMP-sialic acid hydroxylase occurred after the Homo-Pan divergence. Proc Natl Acad Sci USA 95: 11751-11756. [Crossref]

8. Chou HH, Hayakawa T, Diaz S, Krings M, Indriati E, et al. (2002) Inactivation of CMP-N-acetylneuraminic acid hydroxylase occurred prior to brain expansion during human evolution. Proc Natl Acad Sci USA 99: 11736-11741.

9. Tangvoranuntakul P, Gagneux P, Diaz S, Bardor M, Varki N, et al. (2003) Human uptake and incorporation of an immunogenic nonhuman dietary sialic acid. Proc Natl Acad Sci USA 100: 12045-12050.

10. Miyake M, Ito M, Hitomi S, Ikeda S, Taki T, et al. (1988) Generation of two murine monoclonal antibodies that can discriminate $\mathrm{N}$-glycolyl and $\mathrm{N}$-acetyl neuraminic acid residues of GM2 gangliosides. Cancer Res 48: 6154-6160. [Crossref]

11. Padler-Karavani V, Yu H, Cao H, Chokhawala H, Karp F, et al. (2008) Diversity in specificity, abundance, and composition of anti-Neu5Gc antibodies in normal humans: potential implications for disease. Glycobiology 18: 818-830. [Crossref]

12. Nguyen DH, Tangvoranuntakul P, Varki A (2005) Effects of natural human antibodie against a nonhuman sialic acid that metabolically incorporates into activated and malignant immune cells. J Immunol 175: 228-236. [Crossref]

13. Miwa Y, Kobayashi T, Nagasaka T, Liu D, Yu M, et al. (2004) Are N-glycolylneuraminic acid (Hanganutziu-Deicher) antigens important in pig-to-human xenotransplantation? Xenotransplantation 11: 247-253. [Crossref]

14. Varki A (2009) Multiple changes in sialic acid biology during human evolution Glycoconj $J$ 26: 231-245. [Crossref]

15. Pham T, Gregg CJ, Karp F, Chow R, Padler-Karavani V, et al. (2009) Evidence for a novel human-specific xeno-auto-antibody response against vascular endothelium. Blood 114: 5225-5235. [Crossref]

16. Samraj AN, Pearce OM, Läubli H, Crittenden AN, Bergfeld AK, et al. (2015) A red meat-derived glycan promotes inflammation and cancer progression. Proc Natl Acad Sci USA 112: 542-547

17. Padler-Karavani V, Tremoulet AH, Yu H, Chen X, Burns JC, et al. (2013) A simple method for assessment of human anti-Neu5Gc antibodies applied to Kawasaki disease. PLoS One 8: e58443. [Crossref]

18. Varki NM, Strobert E, Dick EJ Jr, Benirschke K, Varki A (2011) Biomedical difference between human and nonhuman hominids: potential roles for uniquely human aspects of sialic acid biology. Annu Rev Pathol 6: 365-393. [Crossref]

19. Kutteh WH, Hatch KD, Blackwell RE, Mestecky J (1988) Secretory immune system of the female reproductive tract: I. Immunoglobulin and secretory component-containing cells. Obstet Gynecol 71: 56-60. [Crossref]

20. Dechaud H, Maudelonde T, Daurès JP, Rossi JF, Hédon B (1998) Evaluation of endometrial inflammation by quantification of macrophages, $\mathrm{T}$ lymphocytes, and interleukin-1 and -6 in human endometrium. J Assist Reprod Genet 15: 612-618.

21. Cervera R, Balasch J (2008) Bidirectional effects on autoimmunity and reproduction. Hum Reprod Update 14: 359-366. [Crossref]

22. Ghaderi D, Springer SA, Ma F, Cohen M, Secrest P, et al. (2011) Sexual selection by female immunity against paternal antigens can fix loss of function alleles. Proc Natl Acad Sci USA 108: 17743-17748. [Crossref]

23. Veeck L (1999) Preembryo grading and degree of cytoplasmic fragmentation. Veeck L editor. An Atlas of human gametes and conceptuses: an illustrated reference for assisted reproductive technologies. Parthenon, New York. CRC Press; 46-51.

24. Sandra L Diaz, VeredPadler-Karavani, Darius Ghaderi, Nancy Hurtado-Ziola, Hai Yu, et al. (2009) Sensitive and Specific Detection of the Non-Human Sialic Acid $\mathrm{N}-$ Glycolylneuraminic Acid In Human Tissues and Biotherapeutic Products. PLoS ONE 4: e4241. [Crossref]

25. Manzi AE, Diaz S, Varki A (1990) High-pressure liquid chromatography of sialic acids on a pellicular resin anion-exchange column with pulsed amperometric detection: comparison with six other systems. Anal Biochem 188: 20-32. [Crossref]

26. Lewis AL, Nizet V, Varki A (2004) Discovery and characterization of sialic acid O-acetylation in group B Streptococcus. Proc Natl Acad Sci USA 101: 11123-11128. [Crossref]

Copyright: (C2015 Sroga JM. This is an open-access article distributed under the terms of the Creative Commons Attribution License, which permits unrestricted use, distribution, and reproduction in any medium, provided the original author and source are credited. 